\title{
METANÁLISIS EN RED: COMPARACIONES INDIRECTAS Y MIXTAS, UN NUEVO MÉTODO AL SERVICIO DE LA EPIDEMIOLOGÍA CLÍNICA Y LA SALUD PÚBLICA
}

\author{
Herney Andrés García-Perdomo ${ }^{1, a}$, Aurelio Tobías ${ }^{2,3, b}$
}

\begin{abstract}
RESUMEN
La presente revisión trata de describir algunos conceptos fundamentales de los metanálisis en red y las comparaciones indirectas y su aplicación en las ciencias clínicas. Actualmente existe un incremento de revisiones sistemáticas y metanálisis para lo que se requiere la existencia de comparaciones directas entre las diferentes intervenciones, sin embargo, en múltiples ocasiones, esto no es posible dada la carencia de evidencia relacionada con todas las comparaciones posibles. De tal manera que se han desarrollado herramientas estadísticas importantes que permiten hacer comparaciones múltiples e indirectas a partir de las ya existentes. Para concluir, los metanálisis en red son una herramienta novedosa que ayuda a los clínicos, investigadores y a los organismos gubernamentales en la toma de decisiones tanto clínicas como de salud pública.
\end{abstract}

Palabras clave: Revisión; Metanálisis; Salud pública (fuente: DeCS BIREME).

\section{NETWORK META-ANALYSIS: MIXED AND INDIRECT TREATMENT COMPARISONS. A NEW METHOD TO THE SERVICE OF CLINICAL EPIDEMIOLOGY AND PUBLIC HEALTH}

\begin{abstract}
This review describes essential concepts of network meta-analysis and indirect comparisons and their application to clinical science. There are an increasing number of systematic reviews and meta-analyses providing direct comparisons between different interventions, although this is often not feasible when there is a lack of evidence related to all possible comparisons. Thus, important statistical tools that help make indirect comparisons based on previously existing comparisons have been developed. Network meta-analyses are an innovative tool that could assist doctors, researchers and governmental organizations when making clinical and public health decisions.
\end{abstract}

Key words: Review; Meta-analysis; Public health (source: MeSH NLM).

\section{INTRODUCCIÓN}

En el ámbito de la síntesis de evidencia, se ha declarado a las revisiones sistemáticas/metanálisis (RS/MA) como herramientas fundamentales para determinar la magnitud del efecto con un adecuado rigor y calidad científica ${ }^{(1-4)}$. Sin embargo, cuando de comparaciones se trata, esta herramienta requiere que existan comparaciones directas entre el medicamento $\mathrm{A}$ y el medicamento $B$, de otra forma, no es posible realizarla y en la actualidad carecemos de experimentos que realicen todas las comparaciones posibles ${ }^{(5)}$
A falta de evidencia directa, se han desarrollado herramientas como el metanálisis en red y los métodos de comparaciones indirectas. Esto permite tener en cuenta todos los estudios disponibles y partir de allí, hacer comparaciones frente a un elemento en común para estimar el efecto de una intervención de manera indirecta $(6,7)$.

El objetivo de la presente revisión es describir los conceptos fundamentales de los metanálisis en red y las comparaciones indirectas, así como su aplicación en las ciencias clínicas.

\footnotetext{
Universidad del Valle. Cali, Colombia. Hospital Universitario del Valle Cali, Colombia.

Instituto de Diagnóstico Ambiental y Estudios del Agua (IDAEA). Barcelona, España.

Centro Superior de Investigaciones Científicas (CSIC). Barcelona, España.

Doctor en Medicina Clínica y Salud Pública.

Doctor en Medicina Preventiva y Salud Pública

Recibido: 03-05-15 Aprobado: 07-10-15
} 


\section{CONCEPTOS FUNDAMENTALES}

El metanálisis permite sintetizar de manera estadística, la evidencia directa de estudios disponibles sobre una pregunta de investigación clínica a partir de una adecuada revisión sistemática ${ }^{(3,8)}$. El metanálisis en red es una herramienta diseñada para evaluar la efectividad comparativa entre tratamientos experimentales con similares características, que no han sido comparados directamente en un experimento clínico. A diferencia de los metanálisis tradicionales, en los cuales se resume la evidencia de experimentos que han evaluado la misma combinación de tratamiento versus otro tratamiento/ placebo, esta nueva herramienta, compara los resultados de dos o más estudios que tienen un punto o un tratamiento en común ${ }^{(9)}$.

El metanálisis en red, también conocido como metanálisis de comparaciones múltiples o metanálisis de comparaciones mixtas, permite obtener apreciaciones indirectas a partir de estudios que incluyen comparaciones y un comparador en común ${ }^{(8)}$. Al conocer los estimados del efecto de $A$ versus $B$ y $B$ versus $C$, se puede establecer las comparaciones de $A$ versus $C$ de manera indirecta. Esto se puede realizar cuando existe ausencia de información que permita conocer el efecto de $A$ versus $C$, de esta forma se establecen comparaciones tipo: $C A$ indirecta $=C B$ directa $-A B$ directa $(5,6,10)$. Las comparaciones indirectas requieren establecer conceptos tales como: la transitividad y la consistencia ${ }^{(6)}$.

Para iniciar, así como en el metanálisis convencional, con el método de las comparaciones indirectas o mixtas, se requiere que los estudios sean comparables en términos de su diseño, la población, la duración del tratamiento, la variable de resultado final, así como las variables que podrían modificar el efecto.

Las condiciones de transitividad y consistencia deben evaluarse en los diferentes metanálisis en red, sin embargo, la transitividad debe ser especialmente evaluada en las comparaciones indirectas, por otro lado, en las comparaciones mixtas, ambos conceptos deben ser evaluados ${ }^{(6)}$.

Con respecto a la transitividad, existen algunas condiciones para determinarla (Figura 1):

1 El comparador común debe ser definido de manera similar cuando aparece en comparaciones directas $A$ versus $B$ y $B$ versus $C$. En algunas ocasiones puede ser permitida cierta "flexibilidad" aunque esta debe ser sustentada con la literatura. La transitividad no se logrará cumplir cuando el comparador común es diferente en las comparaciones directas.

2 En los experimentos $A$ versus $B$ que no tienen brazo o tratamiento $C$, se supone que los brazos ausentes son debidos al azar. La transitividad no se cumplirá si la elección del comparador es asociada con la eficacia relativa de las intervenciones.

3 Los experimentos con comparaciones directas $A$ versus $B$ y $B$ versus $C$ no difieren con respecto a la distribución de posibles variables modificadoras del efecto. En el supuesto que existan tratamientos nuevos y antiguos, en los que algunas variables puedan cambiar en el tiempo, estas podrían ser modificadoras del efecto, por tanto no cumpliría con la condición de transitividad.

4 Los pacientes aleatorizados en las comparaciones directas podrían ser asignados a cualquiera de los tratamientos (A, B ० C). La transitividad no podría cumplirse si los tratamientos no son comparables en teoría y práctica, ni si tienen diferentes indicaciones terapéuticas.

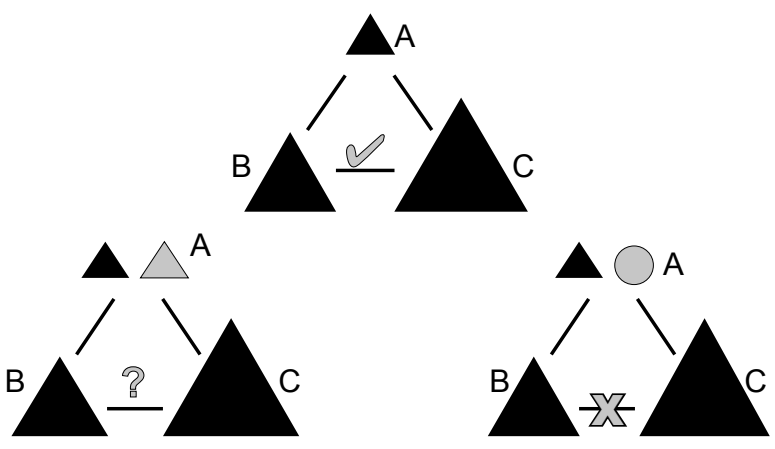

Figura 1. Condición de transitividad de los metanálisis en red

La consistencia se refiere a asumir que la evidencia directa e indirecta son estimaciones del mismo parámetro. Es decir, que si se hubiera incluido el brazo adicional en los experimentos $A$ versus $B$ y $B$ versus $C$, el estimado del efecto debiera ser similar. En otras palabras, no deben existir discrepancias entre el efecto de los tratamientos entre las comparaciones directas e indirectas $\left(C A\right.$ indirecta $=$ CA directa) ${ }^{(11,12)}$. La consistencia puede ser evaluada y comprobada por medio de pruebas estadísticas mediante método de Bucher o factores de inconsistencia ${ }^{(5,11,12)}$ (Figura 2).

\section{Comparación indirecta}

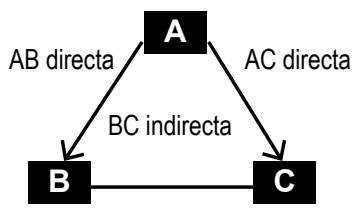

Transitividad

\section{Comparación mixta}

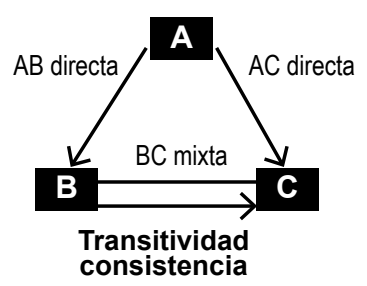

Figura 2. Condición de transitividad y consistencia en las comparaciones indirectas y mixtas. Modificado de CataláLópez F \& Tobías A ${ }^{(6)}$ 


\section{APLICACIÓN CLÍNICA}

En la actualidad, los metanálisis en red han ganado adeptos. Los clínicos, investigadores, editores y agencias de regulación han utilizado artículos con este tipo de métodos como fuente de información e interés para evaluar la eficacia y seguridad de intervenciones. La metodología de comparaciones indirectas también permitiría explorar sesgos por medio de la evaluación de las variables confusoras o modificadoras del efecto. De la misma manera, se podría evaluar una clasificación de los diferentes tratamientos para así determinar la probabilidad de ser el mejor o más efectivo ${ }^{(8)}$.

Para los clínicos, esto podría considerarse para conocer el mejor tratamiento para una condición específica en salud. De igual manera, existe el problema que la respuesta sea dada por una evidencia débil, por este motivo, se debe prestar mayor atención a la magnitud de los efectos y su grado de incertidumbre ${ }^{(9,13,14)}$.

\section{RECOMENDACIONES DE ESCRITURA PARA LOS METANÁLISIS EN RED}

Además de las guías de adecuada conducción y reporte de las revisiones sistemáticas y metanálisis como las elaboradas por Cochrane y PRISMA ${ }^{(15-17)}$, existen algunos elementos fundamentales para describir las revisiones sistemáticas que llevan consigo las comparaciones indirectas y que son fundamentales en el análisis de su reporte ${ }^{(18,19)}$. A continuación se describen de acuerdo con Catalá-López et al. ${ }^{(8)}$ en la Tabla 1.

Tabla 1. Recomendaciones de escritura para los metanálisis en red. Modificado de Catalá-López F. \& Tobías A. ${ }^{(5)}$

\section{Introducción}

¿Está claramente justificada la realización de un metanálisis en red y los objetivos son descritos de forma precisa?

¿Los objetivos son relevantes en la práctica clínica?

\section{Materiales y métodos}

¿La población objetivo, los tratamientos evaluados y la(s) variable(s) de resultado del efecto se especifican claramente?

¿Se tuvo en cuenta la declaración PRISMA para la revisión sistemática?

¿Se describen claramente los métodos de búsqueda, las estrategias de búsqueda complementarias, los criterios de inclusión/ exclusión y los métodos de extracción de datos de los estudios?

¿Se tienen en cuenta la calidad y validez de los artículos siguiendo criterios explícitos y reproducibles?

¿La transitividad es discutida?

¿Los métodos utilizados para los análisis estadísticos incluyendo la justificación del modelo estadístico para comparaciones directas e indirectas se describen claramente?

¿Se presentan estimaciones de comparaciones directas, indirectas o la combinación de ambas?

¿Se presentan los efectos de los tratamientos para cada alternativa evaluada frente al tratamiento de referencia de acuerdo con la red de evidencia definida?

¿Se describen métodos para abordar posibles sesgos?

¿Los métodos para evaluar la heterogeneidad y la consistencia están claramente descritos?

¿Se describe cómo se aplicaron todos estos criterios y si se establecieron a priori?

¿Se presentaron análisis de sensibilidad para valorar el efecto de la incertidumbre?

\section{Resultados}

¿Los resultados de los estudios en una red de evidencia se presentan claramente? ¿Se presenta una figura con la red de evidencia?

¿Se presenta la información de cada estudio por separado?

¿Se presentan los resultados del metanálisis en red de forma clara, objetiva y transparente?

¿Se discuten las limitaciones?

\section{Discusión}

¿Se hace una descripción o resumen de los principales hallazgos?

¿Se discuten las limitaciones del metanálisis en red?

¿Se discuten las consecuencias para la toma de decisiones en la práctica clínica?

¿Se justifican las conclusiones del trabajo? 
Por otro lado, existen una serie de organizaciones que han desarrollado recomendaciones para la realización y escritura de metanálisis en red y las relacionamos a continuación:

- PBAC (Australia, 2008). Indirect Comparisons Working Group Report (http://www.pbs.gov.au/).

- CADTH (Canada, 2009). Indirect Evidence: Indirect Treatment Comparisons in Meta-Analysis (http:// www.cadth.ca/).

- NICE (Reino Unido, 2012). Generalised Linear Modelling Framework for Network Meta-analysis \& Inconsistency in Networks and Evidence Synthesis of Treatment Efficacy (http://www.nicedsu.org.uk/).

- IQWiG (Alemania, 2012). Importance of Results from Indirect Comparisons (http://www.iqwig.de/).

- ISPOR (2011). Indirect Treatment Comparisons and Network Meta-analysis (http://www.ispor.org/).

- EUnetHTA (2013). Comparators \& Comparisons Direct and Indirect Comparisons (http://www. eunethta.eu/).

Dada la amplia variabilidad de guías para un apropiado reporte, un grupo de expertos en revisiones sistemáticas, participaron en una revisión, así como en una encuesta y una serie de discusiones, que le llevaron a delimitar una nueva serie de 32 elementos en forma de lista de chequeo, como extensión de la herramienta PRISMA para el metanálisis en red. Esta herramienta permitirá un adecuado reporte de este nuevo método estadístico, dados los puntos fundamentales que varían ${ }^{(20,21)}$.

\section{LIMITACIONES}

A pesar que las comparaciones indirectas son una herramienta actual e importante a la hora de hacer análisis estadísticos de intervenciones que no se hayan evaluado de forma directa, su uso también tiene riesgos potenciales. Por ejemplo, requiere asumir elementos tales como la transitividad y la consistencia. En la literatura se han publicado artículos que sugieren el incumplimiento de la transitividad entre los diferentes estudios incluidos en el MA o que los resultados de los métodos mixtos no son consistentes. Sin embargo, cabe anotar que los métodos están en desarrollo, aun se aprecia bajo poder estadístico y falta mayor progresión de ellos, pero tienen un futuro muy prometedor ${ }^{(8,22-24)}$. Adicionalmente, los métodos relacionados con comparaciones indirectas o mixtas podrían sobre o subestimar los efectos de los tratamientos, comparado con la evidencia que procede de comparaciones directas ${ }^{(25-27)}$. Aún se requieren mayores avances en las diferentes técnicas estadísticas para aumentar el conocimiento disponible y así incrementar su generalización y aplicación en la toma de decisiones ${ }^{(6)}$.

El metanálisis en red tiene su validez en supuestos. Se requiere que los estudios sean de buena calidad, que no exista variabilidad entre los estudios y se disminuyan los sesgos que pueden existir en las revisiones sistemáticas ${ }^{\left({ }^{(8)} \text {. }\right.}$ Los metanálisis en red deben realizarse en el marco de una revisión sistemática apropiada, rigurosa, en la que se evalúa la red completa de estudios, tratando de evitar cualquier tipo de sesgo ${ }^{(8)}$.

\section{CONCLUSIONES}

Los metanálisis en red son una herramienta novedosa que ayuda a los clínicos, investigadores y a los organismos gubernamentales en la toma de decisiones tanto clínicas como de salud pública. Esta nueva metodología involucra la realización de una excelente, exhaustiva y rigurosa revisión sistemática, que en conjunto servirá como fuente de información, generadora de nuevas ideas tanto en la epidemiología clínica como en la salud pública. Sugerimos mayor entrenamiento en estas técnicas tanto en los lectores, editores, revisores e investigadores para mejorar la calidad de las publicaciones en las revistas biomédicas.

Contribuciones de autoría: HAGP y AT contribuyeron en la concepción y diseño del artículo, recolección, obtención de resultados, análisis e interpretación de datos, así como en la redacción y revisión crítica del artículo, ambos aprobaron la versión final.

Fuentes de financiamiento: autofinanciado.

Conflictos de interés: los autores declaran no tener conflictos de interés.

\section{REFERENCIAS BIBLIOGRÁFICAS}

1. Ferreira González I, Urrútia G, Alonso-Coello P. [Systematic reviews and meta-analysis: scientific rationale and interpretation]. Rev Esp Cardiol. 2011;64(8):688-96. doi: 10.1016/j. recesp.2011.03.029. [Artículo en Español]

2. Moher D, Liberati A, TetzlaffJ, Altman
DG; PRISMA Group. Preferred reporting items for systematic reviews and meta-analyses: the PRISMA statement. PLoS Med. 2009;6(7):1-6.

3. García-Perdomo HA. Síntesis de la evidencia en educación para la salud. Inv Ed Med. 2014;3(12):147-54.

4. García-Perdomo HA. Evidence synthesis and meta-analysis: a practical approach. Int J Urol Nurs. 2015 Jul 28:1-7. Doi: 10.1111/ijun.12087.

5. Catalá-López F, Tobías A. Síntesis de la evidencia clínica y metaanálisis en red con comparaciones indirectas. Med Clin. 2013;140(4):182-7.

6. Catalá-López F, Tobías A. Evidencia 
clínica procedente de comparaciones indirectas y mixtas: algunas consideraciones prácticas. Farm Hosp. 2012;36(6):556-64.

7. Sutton AJ, Higgins JP. Recent developments in meta-analysis. Stat Med. 2008;27(5):625-50.

8. Catalá-López F, Tobías A, Roque M. Conceptos básicos del metaanálisis en red. Aten Primaria. 2014;46(10):57381.

9. Mills EJ, Thorlund K, Ioannidis JPA. Demystifying trial networks and network meta-analysis. BMJ. 2013;346:£2914. doi: 10.1136/bmj.f2914.

10. Bucher H, Guyatt GH, Griffith L, Walter SD. The results of direct and indirect treatment comparisons in meta-analysis of randomized controlled trials. J Clin Epidemiol. 1997;50(6):683-91.

11. Dias $S$, Welton NJ, Caldwell DM, Ades AE. Checking consistency in mixed treatment comparison meta-analysis. Stat Med. 2010;29(7-8):932-44. doi: $10.1002 / \operatorname{sim} .3767$.

12. Salanti G, Higgins JP, Ades AE, Ioannidis JP. Evaluation of networks of randomized trials. Stat Methods Med Res. 2008;17(3):279-301.

13. Trinquart L, Chatellier G, Ravaud P. Adjustment for reporting bias in network meta-analysis of antidepressant trials. BMC Med Res Methodol. 2012;12:150. doi: 10.1186/1471-2288-12-150.

14. Trinquart L, Abbé A, Ravaud P. Impact of reporting bias in network meta-analysis of antidepressant placebo-controlled trials. PLoS One. 2012;7(4):e35219. doi: 10.1371/ journal.pone.0035219.

15. Higgins J, Green S, ed. Cochrane Handbook for Systematic Reviews of Interventions, Version 5.1.0. The Cochrane Collaboration; 2011.

16. Moher D, Liberati A, TetzlaffJ, Altman D. Preferred reporting ítems for systematic reviews and meta-analyses: the PRISMA statement. Ann Intern Med. 2009 Aug 18;151(4):264-9.

17. Swartz MK. The PRISMA statement: A guideline for systematic reviews and meta-analyses. J Pediatr Health Care. 2011;25(1):1-2. doi: 10.1016/j. pedhc.2010.09.006.

18. Bafeta A, Trinquart L, Seror R, Ravaud P. Reporting of results from network meta-analyses: methodological systematic review. BMJ. 2014;348:g1741.

19. Bafeta A, Trinquart L, Seror R, Ravaud P. Analysis of the systematic reviews process in reports of network metaanalyses: methodological systematic review. BMJ. 2013;347:f3675. doi: 10.1136/bmj.f3675.

20. Hutton B, Salanti G, Caldwell DM, Chaimani A, Schmid CH, Cameron $\mathrm{C}$, et al. The PRISMA Extension Statement for Reporting of Systematic Reviews Incorporating Network Metaanalyses of Health Care Interventions: Checklist and Explanations. Ann Intern Med. 2015;162(11):777-84. doi: 10.7326/M14-2385.

21. Hutton B, Salanti G, Chaimani A, Caldwell DM, Schmid C, Thorlund $\mathrm{K}$, et al. The quality of reporting methods and results in network metaanalyses: an overview of reviews and suggestions for improvement. PLoS One. 2014;9(3):e92508. doi: 10.1371/ journal.pone.0092508.

22. Catalá-López F, Hutton B, Moher D. La propiedad transitiva en los ensayos clínicos controlados: si B es mejor que $\mathrm{A}$ y $\mathrm{C}$ es mejor que $\mathrm{B}$, ¿C será mejor que
A?. Rev Esp Cardiol. 2014;67(8):597602.

23. Song F, Xiong T, Parekh-Bhurke S, Loke YK, Sutton AJ, Eastwood AJ, et al. Inconsistency between direct and indirect comparisons of competing interventions: meta-epidemiological study. BMJ. 2011;343:d4909. doi: 10.1136/bmj.d4909.

24. Veroniki AA, Vasiliadis HS, Higgins JP, Salanti G. Evaluation of inconsistency in networks of interventions. Int J Epidemiol. 2013;42(1):332-45. doi: $10.1093 /$ ije/dys222.

25. Chou R, Fu R, Huffman LH, Korthuis PT. Initial highly-active antiretroviral therapy with a protease inhibitor versus a non-nucleoside reverse transcriptase inhibitor: discrepancies between direct and indirect meta-analyses. Lancet. 2006;368(9546):1503-15.

26. Mills EJ, Ghement I, O'Regan C, Thorlund K. Estimating the power of indirect comparisons: a simulation study. PLoS One. 2011;6(1):e16237. doi: 10.1371/journal.pone.0016237.

27. Madan J, Stevenson MD, Cooper KL, Ades AE, Whyte S, Akehurst. Consistency between direct and indirect trial evidence: is direct evidence always more reliable?. Value Health. 2011; 14(6):953-60. doi: 10.1016/j.jval.2011.05.042.

Correspondencia: Herney García.

Dirección: Universidad de Valle, Calle 4B\# 36-00. Cali, Colombia.

Teléfono: (+57)3212195102

Correo electrónico: herney.garcia@correounivalle. edu.co 